\title{
Enumeration of unrooted odd-valent regular planar maps
}

\author{
Zhicheng Gao* \\ Faculty of Business Administration \\ University of Macau \\ Macau \\ China \\ zcgao@umac.mo
}

\author{
Valery A. Liskovets ${ }^{\dagger}$ \\ Institute of Mathematics \\ National Academy of Sciences \\ Minsk, 220072 \\ Belarus \\ liskov@im. bas-net.by
}

\author{
Nicholas Wormald ${ }^{\ddagger}$ \\ Department of Combinatorics and Optimization \\ University of Waterloo \\ Waterloo ON \\ Canada N2L 3G1 \\ nwormald@uwaterloo.ca
}

June 10, 2005

\begin{abstract}
We derive closed formulae for the numbers of rooted maps with a fixed number of vertices of the same odd degree except for the root vertex and one other vertex of degree 1. A similar result, but without the vertex of degree 1, was obtained by the first author and Rahman. These formulae are combined with results of the second author to count unrooted regular maps of odd degree. We succeed in finding, for each even $n$, a closed formula $f_{n}(r)$ for the number of unrooted maps (up to orientation-preserving homeomorphisms) with $n$ vertices and odd degree $r$, provided $r$ is an odd prime or $\operatorname{gcd}(r, n-2)=1$ or $n=2$. The functions $f_{n}$ become more cumbersome as $n$ increases, but for $n>2$ each has a bounded number of terms independent of $r$.
\end{abstract}

2000 Mathematics Subject Classification. Primary: 05C30, Secondary: 05A15.

Key words. odd degree, unrooted map, rooted planar map, regular map, rotation, quotient map, closed formula

\footnotetext{
*Supported by University of Macau.

${ }^{\dagger}$ Supported by the Belarusian RFFR (grant No. F05-227).

${ }^{\ddagger}$ Supported by the Canada Research Chairs program, NSERC and the University of Macau.
} 


\section{Introduction}

\subsection{Motivation}

In the late 1970's the second-named author developed a method of counting unrooted planar maps [Lis81] (see also [Lis85, Lis98]). It relies on the concept of the quotient map of a (geometrically implemented) map with respect to a rotational automorphism. The idea is to reduce the problem to the enumeration of rooted maps of the same type and the arising quotient maps. The method turned out to be effective for diverse classes of maps (see [Lis04]). In particular it is quite applicable for counting unrooted vertex degree specified maps, although the resulting reductive formulae are necessarily fairly cumbersome. But in general we encounter a more serious obstacle in applying this approach: the enumeration of the arising rooted degree specified maps. If the maps under consideration are eulerian, that is even-valent, then all the quotient maps are eulerian or unicursal (the latter term means that only two vertices are of odd degree), and there are remarkable sum-free formulae discovered by Tutte [Tut62] for counting rooted eulerian or unicursal maps with a given vertex degree distribution. In particular, this enabled the second author to count unrooted regular maps of even degree [Lis85].

Sum-free closed formulae are appealing goals for research in enumeration. However, in the general case for maps with odd-degree vertices such simple formulae are not known (see [BenC94], where a certain general but inconvenient formula is given). So the corresponding reductive formulae are impractical for maps having many odd-degree vertices. At present, the only known notable exception is a simple closed formula for the number of rooted 3-regular maps [Mul66]. Accordingly, there is a simple formula for the number of unrooted 3-regular maps. This was published (with a minor error) in [LisW87]. A technique developed by the first author [Gao93] (cf. [GaoR97]) shows that there are other classes of effectively enumerable rooted maps with many odd-degree vertices, namely, $r$-regular maps (see below). However, in order to be applicable for counting unrooted maps of the same classes, the rooted enumeration should be extended to the maps of a similar specification but possessing one or two additional odd-valent vertices. This is one of the main aims of the present paper. It is achieved, with simple closed formulae, if we permit one extra vertex of degree 1 and another of arbitrary degree. Even this requires considerable effort and a nontrivial extension of the technique in [GaoR97] to be able to deal with truncations of power series which somewhat mysteriously cancel with other nice functions (see (3.28)). Further extensions look very difficult.

The other main aim is to translate these results to the enumeration of unrooted regular maps of odd degree. We find closed formulae for the numbers of these maps with a given number $n$ of vertices as a function of the odd degree $r$, provided $r$ satisfies certain restrictions. These restrictions could be removed (and the formula completed) if we could find a similar closed formula for the number of rooted maps with all vertices of degree $r$ except for two vertices of arbitrary (but equal) degree. Our basic approach makes it clear how to obtain non-closed (recursive) formulae for the cases that we omit here, if that is desired. This is a much more routine job than the task we attempt here. 


\subsection{Main definitions and notation}

A planar map is a 2-cell embedding of a planar connected graph (loops and multiple edges allowed) in an oriented sphere. We consider only planar maps, therefore for brevity we will merely call them maps hereafter. A map is rooted if one of its edge-ends (known also as edge-vertex incidence pairs, darts, semi-edges, or "brins" in French) is distinguished as the root. The corresponding vertex is called the root-vertex. It is well known and important that no non-trivial automorphism of a map leaves the root fixed. The degree of a vertex is the number of edge-ends incident with it. This is often called valency in the map enumeration literature.

In this paper, counting unrooted maps means counting up to orientation-preserving homeomorphisms. There is also a method of counting up to all homeomorphisms given by the third author [Wor81], but this is in general very recursive in nature and not so convenient for obtaining closed formulae.

If $\mathcal{K}(m)$ is a class of maps with $m$ edges, then $K^{+}(m)$ denotes the number of unrooted maps in $\mathcal{K}(m)$ and $K(m)$ denotes the number of rooted maps. Hence

$$
K^{+}(m)=|\mathcal{K}(m)|=\sum_{\Gamma \in \mathcal{K}} 1
$$

and it is clear that

$$
K(m)=\sum_{\Gamma \in \mathcal{K}} \frac{2 m}{|\operatorname{Aut}(\Gamma)|},
$$

where $|\operatorname{Aut}(\Gamma)|$ is the order of the automorphism group of the map $\Gamma$. We define $\mathcal{A}\left(1^{a_{1}} 2^{a_{2}} 3^{a_{3}} \cdots ; m\right)$ to be the class of planar maps of the indicated vertex degree specification, that is, with $a_{i}$ vertices of degree $i$; the implied number of edges, $m=$ $\left(a_{1}+2 a_{2}+3 a_{3}+\cdots\right) / 2$, is also recorded in the notation. These maps have

$$
v=\sum a_{i}
$$

vertices and (by Euler's formula)

$$
f=m-v+2
$$

faces. We call maps of such a specification $r$-regular if $a_{i}=0$ for $i \neq r$. Moreover, we need to consider maps in which one or two vertices of degree 1 are distinguished as singular; a map cannot be rooted at a singular vertex. The presence of singular vertices is indicated by the corresponding number of asterisks $(*)$ in the subscript. Singular vertices play the role of axial elements in a rotational automorphism of a covering of the rooted map (see below).

\section{Reductive enumeration of unrooted regular maps}

In this section we relate the number $A^{+}\left(r^{n}\right)=A^{+}\left(r^{n} ; m\right)$ of unrooted $r$-regular maps with $n$ vertices and $m=n r / 2$ edges to the numbers $A\left(r^{n} ; m\right)$ of the corresponding rooted maps, and rooted maps of certain related types. The technique developed in [Lis81, Lis85] will be used; these references should be consulted for further details of the general discussion in this section. 


\subsection{Quotient maps and liftings}

Consider a map $\Gamma$ and its rotational automorphism $\alpha$ of order $\rho>1$. Then $\alpha$ is determined by two axial cells (poles) and the rotation angle $2 \pi \ell / \rho$, where $\ell, 1 \leq \ell<\rho$, is prime to $\rho$. Now there is a unique quotient map $\Delta=\Gamma / \alpha$. It is defined topologically and can be constructed geometrically by cutting the underlying sphere into $\rho$ identical sectors, taking one of them and glueing it into a new sphere. Conversely, given $\rho$ and two arbitrary axial cells, neither of which may be an edge, $\Delta$ is lifted into a unique map $\Gamma^{\prime}$. In order to use this approach for the enumeration of unrooted maps $\Gamma \in \mathcal{K}(m)$, scheme [Lis81, Lis85], one needs first to properly differentiate all possible rotation axes by types to ensure a certain uniformity of the quotient maps and of their liftings. Namely, a classification $W=\{\omega\}$ of the axes is proper if the number of ways to lift a quotient map $\Delta$ back into $\mathcal{K}(m)$ (in other words, this is the multiplicity of the appearances of $\Delta$ as a quotient map) depends only on $\rho$ and the type $\omega$ of the axis with respect to which $\Delta$ has been obtained. We denote this number by $\psi_{\omega}(\rho)$. Further the corresponding classes of quotient maps should be described thoroughly and enumerated as the rooted ones: $K^{+}(m)$ is easily expressed in terms of these numbers multiplied by $\psi_{\omega}(\rho)$ and summed over all possible $\rho$ and $\omega$.

Typically the classification of axes into types according to the nature of the two axial cells (face-face, face-vertex, etc.) suffices. This is indeed true for the present application. Constraints on $\rho$, together with the numbers of the corresponding quotient maps, are described for each type in Table 1 (where, and thereafter, for brevity, (, ) denotes the greatest common divisor). Recall that $m=n r / 2$, and note that $n$ is always even since $r$ is odd.

Table 1: Quotient maps for $\mathcal{A}\left(r^{n} ; m\right)$

\begin{tabular}{|c|l|l|}
\hline Type $\omega$ & Constraint $:=P_{\omega}(\rho)$ & $\#($ rooted quotient maps $):=A[\omega](\rho)$ \\
\hline face - face & $\rho \mid n / 2$ & $A\left(r^{n / \rho} ; \frac{m}{\rho}\right)$ \\
face - vertex & $\rho \mid(r, n-1)$ & $A\left(\left(\frac{r}{\rho}\right)^{1} r^{(n-1) / \rho} ; \frac{m}{\rho}\right)$ \\
face - edge & $\rho=2 \mid(n / 2+1)$ & $A_{*}\left(1^{1} r^{n / 2} ; \frac{m+1}{2}\right)$ \\
vertex - vertex & $\rho \mid(r, n-2)$ & $A\left(\left(\frac{r}{\rho}\right)^{2} r^{(n-2) / \rho} ; \frac{m}{\rho}\right)$ \\
edge - edge & $\rho=2 \mid n / 2$ & $A_{* *}\left(1^{2} r^{n / 2} ; \frac{m}{2}+1\right)$ \\
\hline
\end{tabular}

In order to explain these data we note that the degrees of the axial cells are diminished $\rho$ times in the quotient maps, while the non-axial cells preserve their degrees. On the other hand, $\rho$ non-axial cells turn into one cell, whereas every axial cell of the original map turns into one axial cell of the quotient map. If the axial cell of $\Gamma$ is an edge (in which case $\rho=2$ ), it turns into an edge ending in a special additional vertex of degree 1. This vertex of the quotient map is called singular. A singular vertex is necessarily axial, and the quotient map cannot be rooted at a singular vertex. Finally, the quotient map contains $m / \rho$ edges if it has no singular vertices, and apart from this, 
every singular vertex contributes an additional term of $1 / 2$ to the number of edges of the quotient.

The constraints in the second column of Table 1 arise from the condition that all parameters of the quotient maps are integers. When $\omega$ is face-face, we have $\rho \mid(n, n r / 2)$ and so $\rho \mid n / 2$ since $r$ is odd. When $\omega$ is face-edge, $\rho=2 \mid(n, n r / 2+1 / 2)$ and so $n / 2$ must be odd. The other cases should be obvious. Note that $\omega$ cannot be of the form vertex-edge because $\rho$ would then be 2 (because of the axial edge) but would also have to divide the degree of the axial vertex, which is odd. So this sixth type $\omega$ does not occur for these maps.

Table 2 contains the values of number $\psi_{\omega}(\rho)$ of admissible choices of the axial cells in the quotient maps. The type is defined as in the lifted map. Here $f$ is the number of faces, a function of other parameters as determined by (4). The number of faces of the quotient maps (the fourth column) is significant for calculating $\psi$ only if at least one axial cell is a face.

Table 2: Choice of axial cells in quotient maps for lifting

\begin{tabular}{|c|l|l|}
\hline Type $\omega$ & $\#($ faces $)$ & $\#($ axes choices $):=\psi_{\omega}(\rho)$ \\
\hline face - face & $\frac{f-2}{\rho}+2$ & $\left(\frac{f-2}{\rho}+2\right)\left(\frac{f-2}{\rho}+1\right) / 2$ \\
face - vertex & $\frac{f-1}{\rho}+1$ & $\frac{f-1}{\rho}+1$ \\
face - edge & $\frac{f+1}{2}$ & $\frac{f+1}{2}$ \\
vertex - vertex & & 1 \\
edge - edge & & 1 \\
\hline
\end{tabular}

\subsection{Reduction}

Now we can establish the desired uniform reductive formula for the number of unrooted regular maps.

\section{Theorem 1}

$$
A^{+}\left(r^{n}\right)=\frac{1}{2 m}\left[A\left(r^{n} ; m\right)+\sum_{2 \leq \rho \leq m} \phi(\rho) \sum_{\omega} \delta_{P_{\omega}(\rho)} \psi_{\omega}(\rho) A[\omega](\rho)\right],
$$

where $m=r n / 2, \phi(\rho)=|\{i: 1 \leq i \leq \rho, \operatorname{gcd}(i, \rho)=1\}|$ is the Euler totient function, the second sum is taken over the five axis types represented in the first column of Table 1 , $P_{\omega}(\rho)$ and $A[\omega](\rho)$ are the corresponding constraint and rooted enumerator represented, respectively, in the second and third columns of the same table, the values of $\psi_{\omega}(\rho)$ are taken from the last column of Table 2, where the parameter $f$ is determined by formula (1.1) and $\delta_{P_{\omega}(\rho)}$ is the characteristic function: $\delta_{P}=1$ if the condition $P$ holds and $\delta_{P}=0$ if $P$ is false. 
Proof: Immediately from the foregoing tables and formulae according to the general enumerative scheme for unrooted planar maps [Lis81, Lis85].

It remains to obtain closed enumerative formulae for the functions $A[\omega](\rho)$ appearing in the right-hand side of (2.1). This will be done in the rest of this paper subject to some restrictions on $n$ and $r$. We can state the form of the answer as follows.

Theorem 2 Let $n>2$ be fixed and let d denote $(r-1) / 2$. Then, provided $r$ is not a multiple of an odd prime divisor of $n-2, A^{+}\left(r^{n}\right)$ is expressible as a polynomial in $\left(\begin{array}{c}2 d \\ d\end{array}\right)$ and the quantities $\delta_{i \mid r}\left(\begin{array}{c}2\lfloor d / i\rfloor \\ \lfloor d / i\rfloor\end{array}\right)$ (for all $i$ dividing $\left.n-1\right)$ with coefficients rational functions of $d$. (For each odd prime divisor $p$, the extra quantity $\delta_{r=p}$ must also be included.) For $n=2$,

$$
A^{+}\left(r^{2}\right)=\frac{1}{2}\left(\begin{array}{c}
2 d \\
d
\end{array}\right)+\frac{1}{2(2 d+1)} \sum_{1 \leq \rho \mid 2 d+1} \phi(\rho)\left(\begin{array}{c}
2\lfloor d / \rho\rfloor \\
d / \rho\rfloor
\end{array}\right)^{2} .
$$

A list of explicit formulae for $A^{+}\left(r^{n}\right)$ for all $n \leq 10$ (and the appropriate $r$ ) is given in Section 4.

\section{Near-regular maps with a degree 1 vertex}

This section deals with rooted maps which have all but the root vertex of degree $r$, and one other vertex of degree 1. These are important for evaluating formula (2.1).

We immediately switch to the duals of the maps to be more in line with the arguments in [GaoR97]. Let $M_{k, m}^{\{r\}}$ denote the number of rooted maps with all internal faces of degree $r$, with $k$ faces altogether (including the unbounded face), and $m$ edges. Similarly, $M_{k, m}^{\{r\}[h]}$ denotes the number of rooted maps with all internal faces of degree $r$ except for one distinguished face of degree $h$, with $k$ faces altogether (including the unbounded face), and $m$ edges. From all these notations, when $r$ is understood it can be omitted; thus $M_{k, m}^{[h]}=M_{k, m}^{\{r\}[h]}$; but if $r$ has different values in two parts of the same formula it will be included.

\subsection{Reduction to $M_{k, m}$ and $M_{k, m}^{[1]}$}

We need to express the entries $A[\omega](r)$ in Table 1 in terms of $M_{k, j}$ and $M_{k, j}^{[1]}$. In the following we use $d=2 r+1$ as before. For all but the fourth type (vertex-vertex), we have (see justification below)

$$
\begin{aligned}
\left.A\left(r^{n / \rho} ; m / \rho\right)\right) & =M_{n / \rho, m / \rho}=M_{k, k d+k / 2} \quad(k=n / \rho) \\
A\left((r / \rho)^{1} r^{(n-1) / \rho} ; m / \rho\right) & =n M_{1+(n-1) / \rho, m / \rho} \\
& =n M_{k, k d-d+(k+r / \rho-1) / 2} \quad(k=1+(n-1) / \rho) \\
A_{*}\left(1^{1} r^{n / 2} ;(m+1) / 2\right) & =M_{1+n / 2,(m+1) / 2}^{[1]} \\
& =M_{k, k d-d+k / 2}^{[1]} \quad(k=1+n / 2), \\
A_{* *}\left(1^{2} r^{n / 2} ; m / 2+1\right) & =(r n / 4) M_{2+n / 2,(m+2) / 2}^{[1]} \\
& =(r n / 4) M_{k, k d-2 d+k / 2}^{[1]} \quad(k=2+n / 2) .
\end{aligned}
$$


For the vertex-vertex type, if $\rho=r$ and $n>2$

$$
\begin{aligned}
A\left((r / \rho)^{2} r^{(n-2) / \rho} ; m / \rho\right) & =(n / 2) M_{2+(n-2) / r, n / 2}^{[1]} \\
& =(n / 2) M_{k, k d-2 d+k / 2}^{[1]} \quad(k=2+(n-2) / r)
\end{aligned}
$$

and if $n=2$

$$
A\left((r / \rho)^{2} r^{(n-2) / \rho} ; m / \rho\right)=M_{2, m / \rho}^{\{r / \rho\}},
$$

whilst in any other case for the vertex-vertex type we have not derived an appropriate formula. Thus, we do not obtain a final result if $n>2$ and $\operatorname{gcd}(r, n-2)$ has any factors (which are potential values of $\rho$ ) other than 1 and $r$. The numbers $n$ for which this can happen are those for which $n-2$ is not a power of 2 . In these cases, since $r$ is odd, the required condition can be restated as

$$
r \text { is an odd prime or } \operatorname{gcd}(r, n-2)=1 \text { or } n=2 \text {. }
$$

To justify equations (3.1) - (3.6), we consider the duals of the maps counted by the $M$ 's. Then faces of degree $r$ become vertices of degree $r$. The equation for the first type is immediate. For the second, the quotient map has $n r / \rho$ rootings, of which $r / \rho$ are on the singular vertex, making a $n$-to- 1 correspondence between the maps required and the ones counted by $M_{1+(n-1) / \rho, n / \rho}$ (where the vertex of degree 1 must be the root vertex). For the third type, we could have used $M$ with a similar adjustment, but by using $M^{[1]}$ no adjustment is required. For the fourth type, $M^{[1]}$ counts maps with the root at a vertex of degree 1, so the adjustment factor is the number of possible rootings of the quotient map, divided by 2 (as there are two vertices of degree 1 ). As the quotient map has $n r / 2 \rho=n / 2$ edges, the factor is $n / 2$. For the fifth case, the argument is similar but the special vertices disallow two possible rootings in the maps counted by $A_{* *}$.

\subsection{The form of $M_{k, m}$ and $M_{k, m}^{[1]}$}

Closed formulae for the numbers $M_{k, m}$ were computed in [GaoR97], so for evaluating $(3.1)-(3.6)$ the only new results required are for $M_{k, m}^{[1]}$. It is nice to know what kind of functions will appear in an argument. In particular, the generating function for rooted plane trees is well known:

$$
M_{1}(x)=M_{1}^{\{r\}}(x)=\frac{1-\sqrt{1-4 x}}{2 x} .
$$

We will work with the set $\mathcal{S}[X]$ of polynomials in $X$ and $X^{-1}$ whose coefficients are rational functions of $d$. It follows easily from the equations in [GaoR97] that

$$
M_{k}(x) X^{1 / 2} x^{-(k-1) d}\left(\begin{array}{c}
2 d \\
d
\end{array}\right)^{1-k} \in \mathcal{S}[X]
$$

where

$$
X=(1-4 x)^{-1}
$$

For $M_{k}^{[1]}(x)$ we will prove a similar result. 
Lemma 1 For $k \geq 2$,

$$
M_{k}^{[1]}(x) X^{-1 / 2} x^{-(k-2) d}\left(\begin{array}{c}
2 d \\
d
\end{array}\right)^{2-k} \in \mathcal{S}[X] .
$$

This lemma is proved at the end of this section, along with the specific calculation of $M_{k}^{[1]}(x)$.

The following result is obtained directly from (3.9) and Lemma 1 by considering the extraction of the appropriate coefficient of $x$. Note that the coefficient of $x^{i}$ in any half-integer power of $X$ is a polynomial in $\left(\begin{array}{c}2 d \\ d\end{array}\right)$ with coefficients being rational functions of $d$. Details of this kind of calculation appear in Section 3.7.

Corollary 1 For each fixed $k, M_{k, k d+k / 2}, M_{k, k d-d+k / 2}^{[1]}$ and $M_{k, k d-2 d+k / 2}^{[1]}$ are all polynomials in $\left(\begin{array}{c}2 d \\ d\end{array}\right)$ with coefficients being rational functions of $d$, and $M_{k, k d-d+t}$ is a polynomial in $\left(\begin{array}{c}2 d \\ d\end{array}\right)$ and $\left(\begin{array}{c}2 t \\ t\end{array}\right)$ with coefficients being rational functions of $d$ and $t$.

Proof of Theorem 2: Immediately from Corollary 1, (3.1) - (3.6) and Theorem 1.

\subsection{A recursion for $M_{k}^{[h]}(x)$}

Our point of access is a recursive type relation analogous to [GaoR97, Lemma 1].

Notation For $i \geq 0$ and a generating function $A(x)=\sum_{m \geq 0} c_{m} x^{m}$, let $\mathbf{R}_{i}(A(x))$ denote the part of $A(x)$ consisting of terms with exponents at least $i$, that is

$$
\mathbf{R}_{i}(A(x))=\sum_{m \geq i} c_{m} x^{m}
$$

Lemma 2 For $k \geq 3$ we have

$$
M_{k}^{[h]}(x)=\frac{x}{\sqrt{1-4 x}}\left(2 \sum_{j=2}^{k-1} M_{j}(x) M_{k+1-j}^{[h]}(x)+\mathbf{R}_{a / 2}\left(M_{k-1}^{[h]}(x)\right)+\mathbf{R}_{(a+\delta) / 2}\left(M_{k-1}(x)\right)\right)
$$

where $a=(k-2) r+h-1$ and $\delta=1$ if $h=1$ and 0 otherwise.

Proof: The common degree $r$ is constant in the following argument. Using the type of decomposition in [GaoR97], let $M$ be a map of the type counted by $M_{k}^{[h]}(x)$, with $m$ edges. There are two cases if the root edge of $M$ is deleted. (As usual for such arguments, the new map(s) resulted are rooted in canonical ways.)

In the first case, two maps result. If the one containing the distinguished face has $j$ faces then the other has $k+1-j$ faces. The generating function for these is

$$
2 x M_{j}(x) M_{k+1-j}^{[h]}(x) .
$$

In the second case, deleting the root edge merges the adjacent face with the root face, and there are two subcases. In the first subcase, the adjacent face has degree $r$, in which case the number of possibilities for the map produced is $M_{k-1, m-1}^{[h]}$, and in the 
second, the adjacent face is the distinguished one of degree $h$, so the new map is counted by $M_{k-1, m-1}$. However, in the first of these cases the degree of the root face of $M$ must be at least 1 , implying (summing the face degrees) that $2 m \geq(k-2) r+h+1$, and so $m-1 \geq((k-2) r+h-1) / 2$. Conversely, for $m-1$ in this range, the reverse operation can be carried out in a unique way. On the other hand, in the second case, we obtain the same constraint if $h \geq 2$, but the stronger constraint $m-1 \geq((k-2) r+h) / 2$ in the case $h=1$ since the degree of $M$ must be at least 2 when the root edge encompasses a face of degree 1 . (For $k=2$, if it had been permitted, the second case would have the same restriction as the first one.) Thus

$$
M_{k}^{[h]}(x)=\sum_{j=1}^{k} 2 x M_{j}(x) M_{k+1-j}^{[h]}(x)+x \sum_{m \geq((k-2) r+h-1) / 2}\left(M_{k-1, m}^{[h]} x^{m}+M_{k-1, m} x^{m}\right)
$$

and the lemma follows upon solving for $M_{k}^{[h]}$ and using (3.8) to simplify the leading factor.

In further work we consider only the case $h=1$. Since the number of edges in maps counted by $M_{k-1}(x)$ is at least $((k-2)(2 d+1)+1) / 2=(a+1) / 2$, the following is immediate.

\section{Corollary 2}

$$
M_{k}^{[1]}(x)=\frac{x}{\sqrt{1-4 x}}\left(2 \sum_{j=2}^{k-1} M_{j}(x) M_{k+1-j}^{[1]}(x)+\mathbf{R}_{a / 2}\left(M_{k-1}^{[1]}(x)\right)+M_{k-1}(x)\right) .
$$

Now for some computations for small values of $k$. As a foundation in addition to (3.8) we use the following formula of Tutte [Tut62] (see also equation (1) in [GaoR97]) for just two faces, the interior one of degree $r$ (even or odd):

$$
M_{2, m}^{\{r\}}=\frac{m-\lfloor r / 2\rfloor}{2 m}\left(\begin{array}{c}
2 m-2\lfloor r / 2\rfloor \\
m-\lfloor r / 2\rfloor
\end{array}\right)\left(\begin{array}{c}
2\lfloor r / 2\rfloor \\
\lfloor r / 2\rfloor
\end{array}\right) \text {. }
$$

We also make use, in one of our alternative derivations of $M_{3}^{[1]}$, of Tutte's formula in the case that the non-root face degree is even:

$$
M_{k, m}^{\{2 d\}}=\frac{(m-1) !(m-(k-1) d)}{(k-1) !(m-k+2) !}\left(\begin{array}{c}
2 m-2(k-1) d \\
m-(k-1) d
\end{array}\right)\left(\begin{array}{c}
2 d-1 \\
d
\end{array}\right)^{k-1} .
$$

For later use, note that (3.11) implies for the generating function (see [GaoR97, Equation 13])

$$
M_{2}(x)=\left(\begin{array}{c}
2 d \\
d
\end{array}\right) \sum_{m \geq d+1} \frac{m-d}{2 m}\left(\begin{array}{c}
2 m-2 d \\
m-d
\end{array}\right) x^{m}
$$

and hence

$$
M_{2}^{\prime}(x)=\left(\begin{array}{c}
2 d \\
d
\end{array}\right) \frac{x^{d}}{(1-4 x)^{3 / 2}} .
$$


From [GaoR97] we also have

$$
M_{3}(x)=\left(\begin{array}{c}
2 d \\
d
\end{array}\right)^{2} x^{2 d+2}(1-4 x)^{-3 / 2} .
$$

We henceforth assume that $r$ is an odd integer, and write $d=(r-1) / 2$ so that $r=2 d+1$. The value of $a / 2$ in Lemma 2 is then, in the case $h=1$, given by

$$
a / 2=d(k-2)+k / 2-1 .
$$

Formulae such as (3.14) and (3.15) we will call closed since they are simple explicit formulae for the generating functions. In the present section, more specifically, a closed expression means that it is a sum of terms involving the power of $x$ and $(1-4 x)$ where the number of terms depends only on the number of vertices, not on $d$. In general, our aim would be to find such closed expressions for $M_{k}^{[h]}$, though we only succeed in the case $h=1$. For the next two calculations, we explore a direct combinatorial argument rather than the recursion in Lemma 2. (However, note that we will later develop a general recursion that also covers the case of $M_{3}^{[1]}$.)

\section{$3.4 M_{2}^{[1]}$}

Start with a rooted plane tree with $m-1$ edges and insert a loop into any of its $2 m-2$ corners, or insert the loop into the root corner and transfer the rooting onto the loop. This shows that

$$
M_{2, m}^{[1]}=(2 m-1) M_{1, m-1}
$$

as $M_{1, m}$ is the number of rooted planar trees with $m$ edges. Thus, using (3.8), we have

$$
M_{2}^{[1]}(x)=\frac{1}{2 \sqrt{1-4 x}}-\frac{1}{2}
$$

regardless of $r$.

\section{$3.5 M_{3}^{[1]}$}

A map $\Gamma$ of this sort can be obtained by inserting a loop into another map, $\Gamma^{\prime}$. For the loop to be inserted into the unbounded face, there are $2 m-2-r$ corners available, and we also permit the possibility of shifting the rooting onto the loop if the root corner is used. In this case $\Gamma^{\prime}$ is counted by $M_{2, m-1}=M_{2, m-1}^{\{r\}}$. For the loop to be inserted in the interior face, there are $r-1$ corners as $\Gamma^{\prime}$ is counted by $M_{2, m-1}^{\{r-1\}}$. Hence

$$
M_{3, m}^{\{r\}[1]}=(2 m-r-1) M_{2, m-1}^{\{r\}}+(r-1) M_{2, m-1}^{\{r-1\}} .
$$

Recalling that $r=2 d+1$ we may apply (3.11) and (3.12) to obtain

$$
\begin{aligned}
M_{3, m}^{[1]} & =\left(\begin{array}{c}
2 d \\
d
\end{array}\right)\left(\begin{array}{c}
2 m-2 d-2 \\
m-d-1
\end{array}\right)\left(\frac{(m-1-d)^{2}}{m-1}+\frac{d(m-d-1)}{m-1}\right) \\
& =(m-d-1)\left(\begin{array}{c}
2 d \\
d
\end{array}\right)\left(\begin{array}{c}
2 m-2 d-2 \\
m-d-1
\end{array}\right) .
\end{aligned}
$$


Note that, comparing with (3.11), we have

$$
M_{3}^{[1]}(x)=2 x^{2} M_{2}^{\prime}(x),
$$

a relation which it would be nice to derive via a direct bijection between the maps counted by $M_{3}^{[1]}(x)$ and those counted by $M_{2}^{\prime}(x)$ (with one less edge and with a distinguished corner). Unfortunately, simply saying that the loop is inserted into the distinguished corner does not apply, since the loop cannot be inserted into a corner in the interior face without destroying the degree condition, and also the $M_{3, n}$ maps with a root on the loop must be produced somehow.

\section{6 $M_{4}^{[1]}$}

The general method in the next Section is quite complicated so we introduce it by dealing with the case of $M_{4}^{[1]}$ separately using a very related approach. From Corollary 2 and using (3.16),

$$
M_{4}^{[1]}(x)=\frac{x}{\sqrt{1-4 x}}\left(\sum_{j=2}^{3} 2 M_{j}(x) M_{5-j}^{[1]}(x)+\mathbf{R}_{2 d+1}\left(M_{3}^{[1]}(x)\right)+M_{3}(x)\right) .
$$

Of the terms in the summation,

$$
2 M_{2}(x) M_{3}^{[1]}(x)=2 x^{2}\left(\left(M_{2}(x)\right)^{2}\right)^{\prime}
$$

by (3.19). Also by (3.15) and (3.17)

$$
2 M_{3}(x) M_{2}^{[1]}(x)=2\left(\begin{array}{c}
2 d \\
d
\end{array}\right)^{2} x^{2 d+2}(1-4 x)^{-3 / 2}\left(\frac{1}{2 \sqrt{1-4 x}}-\frac{1}{2}\right) .
$$

For the next term, note that, using (3.19),

$$
\begin{aligned}
\mathbf{R}_{2 d+1}\left(M_{3}^{[1]}(x)\right) & =\mathbf{R}_{2 d+1}\left(2 x^{2} M_{2}^{\prime}(x)\right) \\
& =2 x^{2} \mathbf{R}_{2 d-1}\left(M_{2}^{\prime}(x)\right) \\
& =2 x^{2}\left(\mathbf{R}_{2 d}\left(M_{2}(x)\right)\right)^{\prime} \\
& =2 x^{2}\left(\mathbf{R}_{2 d+1}\left(M_{2}(x)\right)+x^{2 d} M_{2,2 d}\right)^{\prime} .
\end{aligned}
$$

Adding (3.21) and (3.23) using (3.11) gives

$$
2 x^{2}\left(\left(M_{2}(x)\right)^{2}+\mathbf{R}_{2 d+1}\left(M_{2}(x)\right)\right)^{\prime}+4 d x^{2 d+1}\left(\begin{array}{c}
2 d \\
d
\end{array}\right)^{2} \frac{1}{4} .
$$

It is shown in [GaoR97] just after (18) that

$$
\left(M_{2}(x)\right)^{2}+\mathbf{R}_{2 d+1}\left(M_{2}(x)\right)=\left(\begin{array}{c}
2 d \\
d
\end{array}\right)^{2} x^{2 d+1}(1-4 x)^{-1},
$$


and so putting this all together we have from (3.15) and (3.20)

$$
\begin{gathered}
\frac{x}{\sqrt{1-4 x}}\left(2 x^{2}\left(\left(\begin{array}{c}
2 d \\
d
\end{array}\right)^{2} x^{2 d+1}(1-4 x)^{-1}\right)^{\prime}+4 d x^{2 d+1}\left(\begin{array}{c}
2 d \\
d
\end{array}\right)^{2} \frac{1}{4}\right. \\
\left.+2\left(\begin{array}{c}
2 d \\
d
\end{array}\right)^{2} x^{2 d+2}(1-4 x)^{-3 / 2}\left(\frac{1}{2 \sqrt{1-4 x}}-\frac{1}{2}\right)+\left(\begin{array}{c}
2 d \\
d
\end{array}\right)^{2} x^{2 d+2}(1-4 x)^{-3 / 2}\right)
\end{gathered}
$$

which is

$$
\begin{gathered}
\frac{x}{\sqrt{1-4 x}}\left(\begin{array}{c}
2 d \\
d
\end{array}\right)^{2}\left(2 x^{2}\left((2 d+1) x^{2 d}(1-4 x)^{-1}+4 x^{2 d+1}(1-4 x)^{-2}\right)+4 d x^{2 d+1} \frac{1}{4}\right. \\
\left.+2 x^{2 d+2}(1-4 x)^{-3 / 2}\left(\frac{1}{2 \sqrt{1-4 x}}-\frac{1}{2}\right)+x^{2 d+2}(1-4 x)^{-3 / 2}\right)
\end{gathered}
$$

i.e.

$$
\begin{aligned}
& \frac{x^{2 d+2}}{\sqrt{1-4 x}}\left(\begin{array}{c}
2 d \\
d
\end{array}\right)^{2}\left(2 x\left((2 d+1)(1-4 x)^{-1}+4 x(1-4 x)^{-2}\right)+d\right. \\
& \left.\quad+2 x(1-4 x)^{-3 / 2}\left(\frac{1}{2 \sqrt{1-4 x}}-\frac{1}{2}\right)+x(1-4 x)^{-3 / 2}\right)
\end{aligned}
$$

i.e.

$$
\begin{gathered}
\frac{x^{2 d+2}}{\sqrt{1-4 x}}\left(\begin{array}{c}
2 d \\
d
\end{array}\right)^{2}\left(2 x\left((2 d+1)(1-4 x)^{-1}+4 x(1-4 x)^{-2}\right)+d\right. \\
\left.+x(1-4 x)^{-2}\right)
\end{gathered}
$$

i.e.

$$
M_{4}^{[1]}(x)=\frac{x^{2 d+2}}{\sqrt{1-4 x}}\left(\begin{array}{c}
2 d \\
d
\end{array}\right)^{2}\left(d+2 x(2 d+1)(1-4 x)^{-1}+x(8 x+1)(1-4 x)^{-2}\right) .
$$

\subsection{Higher $M_{k}^{[1]}$}

In the formula for $M_{5}^{[1]}$ from Lemma 2 we have closed expressions plus

$$
2 M_{2}(x) M_{4}^{[1]}(x)+2 M_{3}(x) M_{3}^{[1]}(x)+\mathbf{R}_{3 d+3 / 2}\left(M_{4}^{[1]}(x)\right) .
$$

The middle term is closed. Now in general

$$
2 M_{2} f(x)+\mathbf{R}_{d}(f(x))
$$

is closed when $f(x)=(1-4 x)^{-\alpha}$ for $\alpha$ a half-integer. To be precise, we will calculate $H_{j}(x)$ for integers $j \geq 1$ such that

$$
\mathbf{F}\left((1-4 x)^{-j-1 / 2}\right)=-\left(\begin{array}{c}
-j-3 / 2 \\
d-1
\end{array}\right)(-4 x)^{d} H_{j}(x)
$$


where $\mathbf{F}$ is the linear operator defined as

$$
\mathbf{F}(f(x))=2 M_{2}(x) f(x)+\mathbf{R}_{d} f(x) .
$$

First, differentiating (3.24) we have

$$
\begin{aligned}
2 M_{2}(x) M_{2}^{\prime}(x)+\mathbf{R}_{2 d}\left(M_{2}^{\prime}(x)\right) & =\left(\begin{array}{c}
2 d \\
d
\end{array}\right)^{2}\left(x^{2 d+1}(1-4 x)^{-1}\right)^{\prime} \\
& =\left(\begin{array}{c}
2 d \\
d
\end{array}\right)^{2}\left((2 d+1) x^{2 d}(1-4 x)^{-1}+4 x^{2 d+1}(1-4 x)^{-2}\right) .
\end{aligned}
$$

So using (3.14) and dividing by $\left(\begin{array}{c}2 d \\ d\end{array}\right) x^{d}$, and then using $\left(\begin{array}{c}-5 / 2 \\ d-1\end{array}\right)(-4)^{d}=\frac{-2 d(2 d+1)}{3}\left(\begin{array}{c}2 d \\ d\end{array}\right)$, this determines $H_{1}$ in $(3.28)$ :

$$
H_{1}(x)=\frac{3(2 d+1-8 d x)}{2 d(2 d+1)(1-4 x)^{2}}
$$

Next, note that

$$
\mathbf{R}_{d} x(1-4 x)^{-j-3 / 2}=x \mathbf{R}_{d-1}(1-4 x)^{-j-3 / 2}=x \mathbf{R}_{d}(1-4 x)^{-j-3 / 2}+x\left(\begin{array}{c}
-j-3 / 2 \\
d-1
\end{array}\right)(-4 x)^{d-1}
$$

and hence

$$
\begin{aligned}
\mathbf{F}\left(x(1-4 x)^{-j-3 / 2}\right) & =x 2 M_{2}(x)(1-4 x)^{-j-3 / 2}+\mathbf{R}_{d} x(1-4 x)^{-j-3 / 2} \\
& =x \mathbf{F}\left((1-4 x)^{-j-3 / 2}\right)+x\left(\begin{array}{c}
-j-3 / 2 \\
d-1
\end{array}\right)(-4 x)^{d-1} .
\end{aligned}
$$

So, using linearity of $\mathbf{F}$, for all integers $j$,

$$
\begin{aligned}
\left(\begin{array}{c}
-j-3 / 2 \\
d-1
\end{array}\right)(-4 x)^{d} H_{j}(x) & =-\mathbf{F}\left((1-4 x)^{-j-1 / 2}\right) \\
& =-\mathbf{F}\left((1-4 x)(1-4 x)^{-j-3 / 2}\right) \\
& \left.=-\mathbf{F}\left((1-4 x)^{-j-3 / 2}\right)+4 \mathbf{F}\left(x(1-4 x)^{-j-3 / 2}\right)\right) \\
& =-(1-4 x) \mathbf{F}\left((1-4 x)^{-j-3 / 2}\right)+4 x\left(\begin{array}{c}
-j-3 / 2 \\
d-1
\end{array}\right)(-4 x)^{d-1} \\
& =\left[(1-4 x)\left(\begin{array}{c}
-j-5 / 2 \\
d-1
\end{array}\right) H_{j+1}(x)-\left(\begin{array}{c}
-j-3 / 2 \\
d-1
\end{array}\right)\right](-4 x)^{d} .
\end{aligned}
$$

Writing

$$
B(x, y)=\left(\begin{array}{c}
x-1 / 2 \\
d+y
\end{array}\right)
$$

we may rewrite the equation above as

$$
H_{j}(x)=X^{-1} \frac{B(-j-2,-1)}{B(-j-1,-1)} H_{j+1}(x)-1
$$


with $X$ as in (3.10). Now it is easy to check that for all integers $p$ and $q$

$$
\frac{B(p-1, q)}{B(p, q)}=\frac{2 p-1-2 d-2 q}{2 p-1}
$$

and thus

$$
H_{j+1}(x)=X \frac{2 j+3}{2 j+2 d+1}\left(H_{j}(x)+1\right) .
$$

Starting with (3.30), which may be rewritten as

$$
H_{1}=\frac{X(X+2 d)}{2 d(2 d+1)}
$$

we may use this recurrence to compute $H_{j}$ recursively for all fixed integers $j$, both positive and negative. Moreover, we may write

$$
H_{j}(x)=\tilde{H}_{j}(X)
$$

where $\tilde{H}_{j}$ is a polynomial in $X$ and $1 / X$ whose coefficients are rational functions of $d$ with a bounded number of terms for fixed $j$. The resulting recurrence for $\tilde{H}_{j}$ can be solved using standard techniques to give

$$
\tilde{H}_{j}=\frac{(3 / 2)_{d-1}}{(j+3 / 2)_{d-1}} X^{j} \tilde{H}_{0}+\frac{1}{(j+3 / 2)_{d-1}} \sum_{i=0}^{j-1}(i+3 / 2)_{d-1} X^{j-i}
$$

where $\tilde{H}_{0}=X /(2 d)$, and $(a)_{b}=a(a+1) \cdots(a+b-1)$ is the rising factorial. We may check that (3.34) is also valid for negative integers $j$, in which case the summation symbol is interpreted as $-\sum_{i=j}^{-1}$.

We also need to truncate at some other places. For $b \geq 0$, define

$$
\mathbf{F}_{b}(f(x))=2 M_{2}(x) f(x)+\mathbf{R}_{b} f(x)
$$

(so in particular, $\mathbf{F}=\mathbf{F}_{d}$ ). To simplify things a little, let us define

$$
\tilde{M}_{k}^{[1]}(x)= \begin{cases}M_{k}^{[1]}(x) & \text { for } k>2 \\ M_{2}^{[1]}(x)+\frac{1}{2}=\frac{1}{2 \sqrt{1-4 x}} & \text { for } k=2 .\end{cases}
$$

Then the lone term $M_{k-1}(x)$ in Corollary 2 is amalgamated into the term in the summation for $j=k-1$, and we have for $k \geq 3$

$$
\begin{aligned}
M_{k}^{[1]}(x) & =\frac{x}{\sqrt{1-4 x}}\left(2 \sum_{j=2}^{k-1} M_{j}(x) \tilde{M}_{k+1-j}^{[1]}(x)+\mathbf{R}_{a / 2}\left(\tilde{M}_{k-1}^{[1]}(x)\right)\right) \\
& =\frac{x}{\sqrt{1-4 x}}\left(2 \sum_{j=3}^{k-1} M_{j}(x) \tilde{M}_{k+1-j}^{[1]}(x)+\mathbf{F}_{d(k-2)+k / 2-1}\left(\tilde{M}_{k-1}^{[1]}(x)\right)\right)
\end{aligned}
$$

by (3.16). 
From (3.34) we have for fixed $j$ that

$$
H_{j}(x) \in \mathcal{S}[X]
$$

where $\mathcal{S}$ is defined as in Section 3.2. It is easy to check that

$$
B(0,0)=(-4)^{-d}\left(\begin{array}{c}
2 d \\
d
\end{array}\right)
$$

and thus from (3.32), and a similar formula for $B(p, q-1) / B(p, q)$, for any fixed integers $p$ and $q$

$$
(-4)^{d} B(p, q) /\left(\begin{array}{c}
2 d \\
d
\end{array}\right) \text { is a rational function of } d
$$

and hence by (3.28)

$$
\mathbf{F}\left((1-4 x)^{-j-1 / 2}\right) \in\left(\begin{array}{c}
2 d \\
d
\end{array}\right) x^{d} \mathcal{S}[X]
$$

for each fixed $j$.

Proof of Lemma 1 We claim that (3.37) implies by induction that for $k \geq 2$

$$
L_{k}(X):=\tilde{M}_{k}^{[1]}(x) X^{-1 / 2} x^{-(k-2) d} \in\left(\begin{array}{c}
2 d \\
d
\end{array}\right)^{k-2} \mathcal{S}[X],
$$

which immediately implies the lemma.

This is true for $k=2$ by (3.17). Assume $L_{k-1}(X) \in\left(\begin{array}{c}2 d \\ d\end{array}\right)^{k-3} \mathcal{S}[X]$. Then

$$
\mathbf{F}_{d(k-2)+k / 2-1}\left(\tilde{M}_{k-1}^{[1]}(x)\right)=x^{(k-3) d} \mathbf{F}_{d+k / 2-1}\left(L_{k-1}(X) X^{1 / 2}\right) .
$$

This can be evaluated by noticing that $\mathbf{F}_{d+k / 2-1}$ is linear and also

$$
\mathbf{F}_{d+k / 2-1}\left(X^{j+1 / 2}\right)=\mathbf{F}_{d}\left(X^{j+1 / 2}\right)-\sum_{i=d}^{d+\lfloor k / 2-3 / 2\rfloor} x^{i}\left[x^{i}\right] X^{j+1 / 2}
$$

where square brackets denote the extraction of a coefficient. Thus by (3.28)

$$
\mathbf{F}_{d(k-2)+k / 2-1}\left(\tilde{M}_{k-1}^{[1]}(x)\right)=x^{(k-3) d} \sum_{j}-Q(j, k)\left[X^{j}\right] L_{k-1}(X)
$$

where

$$
\begin{aligned}
Q(j, k) & =\left(\begin{array}{c}
-j-3 / 2 \\
d-1
\end{array}\right)(-4 x)^{d} H_{j}(x)+\sum_{i=d}^{d+\lfloor k / 2-3 / 2\rfloor} x^{i}\left[x^{i}\right] X^{j+1 / 2} \\
& =(-4 x)^{d} B(-j-1,-1) \tilde{H}_{j}(X)+\sum_{i=0}^{\lfloor k / 2-3 / 2\rfloor}(-4 x)^{d+i} B(-j, i) \\
& \in x^{d}\left(\begin{array}{c}
2 d \\
d
\end{array}\right) \mathcal{S}[X]
\end{aligned}
$$

by (3.39) and inverting (3.10) to $x=(1-1 / X) / 4$. We now obtain (3.41) recursively from (3.37), (3.9) and (3.42).

Computational note The coefficients of $L_{k}(X)$ can be obtained from the equations referred to in the last line of the above proof together with (3.38), (3.32), (3.34) and (3.33). This gives the value of $M_{k}^{[1]}$ for $k \geq 3$ by the definition of $L_{k}$ in (3.41). 


\section{Results}

First, for completeness, we give the formulae for $M_{k}(x)$ computed using the equations in [GaoR97], which clearly demonstrate the general form of these functions. Note that the coefficients of $M_{2}(x)$ are given in (3.11) and we do not have a closed formula for the generating function.

$$
\begin{gathered}
M_{3}(x)=\frac{1}{16}\left(\begin{array}{c}
2 d \\
d
\end{array}\right)^{2} \frac{(X-1)^{2} x^{2 d}}{\sqrt{X}} \\
M_{4}(x)=\frac{1}{64}\left(\begin{array}{c}
2 d \\
d
\end{array}\right)^{3} \frac{(X-1)^{2}(2 d+X-1) x^{3 d}}{\sqrt{X}} \\
M_{5}(x)=\frac{1}{3072}\left(\begin{array}{c}
2 d \\
d
\end{array}\right)^{4} \frac{(X-1)^{3}\left(64 d^{2}-16 d+48 X d-18 X+3+15 X^{2}\right) x^{4 d}}{X^{3 / 2}} .
\end{gathered}
$$

Here are results for $M_{k}^{[1]}(x)$ obtained as described at the end of Section 3 . The functions $L_{k}(X)$ defined in (3.41) are easily discerned from these. Recall that $\tilde{M}$ and $M$ are the same except when $k=2$.

$$
\begin{gathered}
\tilde{M}_{2}^{[1]}(x)=\frac{1}{2} \sqrt{X} \\
M_{3}^{[1]}(x)=\frac{1}{8}\left(\begin{array}{c}
2 d \\
d
\end{array}\right) \frac{(X-1)^{2} x^{d}}{\sqrt{X}} \\
M_{4}^{[1]}(x)=\frac{1}{64}\left(\begin{array}{c}
2 d \\
d
\end{array}\right)^{2} \frac{(X-1)^{2}(4 d+3 X-3) x^{2 d}}{\sqrt{X}} \\
M_{5}^{[1]}(x)=\frac{1}{256}\left(\begin{array}{c}
2 d \\
d
\end{array}\right)^{3} \frac{(X-1)^{3}\left(5 X^{2}-6 X+12 X d-4 d+12 d^{2}+1\right) x^{3 d}}{X^{3 / 2}} .
\end{gathered}
$$

Writing $U_{k, d}$ for $M_{k, k d+k / 2}$ as in (3.1), $V_{k, d}$ for $M_{k, k d-d+k / 2}^{[1]}$ as in (3.3), and $W_{k, d}$ for $M_{k, k d-2 d+k / 2}^{[1]}$ as in (3.4) and (3.5), we have from (3.11) and the equations above

$$
\begin{gathered}
U_{2, d}=\left(\begin{array}{c}
2 d \\
d
\end{array}\right)^{2} \\
U_{4, d}=\frac{2}{3} d(2 d+1)\left(\begin{array}{c}
2 d \\
d
\end{array}\right)^{4} \\
U_{6, d}=\frac{1}{20} d^{2}(2 d+1)(36 d-1)\left(\begin{array}{c}
2 d \\
d
\end{array}\right)^{6} \\
U_{8, d}=\frac{1}{315} d^{2}(2 d+1)\left(2048 d^{3}-387 d^{2}+20 d-1\right)\left(\begin{array}{c}
2 d \\
d
\end{array}\right)^{8} \\
\frac{1}{9072} d^{2}(2 d+1)\left(250000 d^{5}-100944 d^{4}+14167 d^{3}-1131 d^{2}+73 d-3\right)\left(\begin{array}{c}
2 d \\
d
\end{array}\right)^{10} . \\
V_{2, d}=\frac{2 d+1}{d+1}\left(\begin{array}{c}
2 d \\
d
\end{array}\right)
\end{gathered}
$$




$$
\begin{gathered}
V_{4, d}=\frac{3}{2} d(2 d+1)\left(\begin{array}{c}
2 d \\
d
\end{array}\right)^{3} \\
V_{6, d}=\frac{5}{24} d^{2}(25 d-1)(2 d+1)\left(\begin{array}{c}
2 d \\
d
\end{array}\right)^{5} \\
V_{8, d}=\frac{7}{720} d^{2}(2 d+1)\left(2401 d^{3}-544 d^{2}+35 d-2\right)\left(\begin{array}{c}
2 d \\
d
\end{array}\right)^{7} . \\
W_{2, d}=1 \\
W_{4, d}=d\left(\begin{array}{c}
2 d \\
d
\end{array}\right)^{2} \\
W_{6, d}=\frac{1}{6} d^{2}(16 d-1)\left(\begin{array}{c}
2 d \\
d
\end{array}\right)^{4} \\
W_{8, d}=\frac{1}{60} d^{2}\left(648 d^{3}-182 d^{2}+15 d-1\right)\left(\begin{array}{c}
2 d \\
d
\end{array}\right)^{6} .
\end{gathered}
$$

The numbers of unrooted maps are now counted using (2.1), Tables 1 and 2, equations (3.1)-(3.6), and the appropriate coefficients of $M_{k}(x)$ and $M_{k}^{[1]}(x)$ as given above. There are also some extra ones to use in the case $n=2$ (see below), as well as

$$
M_{k, k d-d+t}
$$

which arises in (3.2) for $t=(k+(2 d+1) / \rho-1) / 2$. It only occurs when $\rho \mid n-1$ as shown in Table 1 (face-vertex case) and can be computed in each case using the formula for $M_{k}(x)$. The result when $k=2$ is $\frac{t}{2 d+t}\left(\begin{array}{c}2 t \\ t\end{array}\right)\left(\begin{array}{c}2 d \\ d\end{array}\right)$, where $t=((2 d+1) / \rho+1) / 2=\lceil d / \rho\rceil$ since $t$ is an integer. Expressed in terms of $s=\lfloor d / \rho\rfloor=t-1$, this becomes $\frac{4 s+2}{2 d+s+1}\left(\begin{array}{c}2 s \\ s\end{array}\right)\left(\begin{array}{c}2 d \\ d\end{array}\right)$. Note that $s=(2 d+1-\rho) /(2 \rho)$, which makes the first factor $4(2 d+1) /(4 d \rho+2 d+\rho+1)$, and this gives simple expressions for particular $\rho$. For $k=4$ we compute $M_{4,3 d+\lceil d / \rho\rceil+1}$ by expanding the formula given above in powers of $X$ and replacing the coefficient of $X^{i-1 / 2}$ by $(-4)^{s+2} B(i, 2)$, where $B$ is defined in (3.31). This simplifies to

$$
M_{4,3 d+s+2}=\frac{2 d s-d-s}{4 s-2}\left(\begin{array}{c}
2 s \\
s
\end{array}\right)\left(\begin{array}{c}
2 d \\
d
\end{array}\right)^{3} .
$$

In the case $\rho=3$ the above expression for $s$ shows that the first factor is $\left(2 d^{2}-6 d+\right.$ $1) /(4 d-10)$. We do not compute this for arbitrary $k$. It is easy to compute what is required in Maple up to large values of $k$.

For $n=2$ there is a special term arising as in (3.6) coming from the vertex-vertex type, when $\psi=1$ and $P_{\omega}(\rho)$ is $\rho \mid r$. This is $\phi(\rho) M_{2, r / \rho}^{\{r / \rho\}}$. The binomial is evaluated as $\left(\begin{array}{c}r / \rho-1 \\ (r / \rho-1) / 2\end{array}\right)$ using (3.11) (as for $U_{2}$ above but with $r$ replaced by $\left.r / \rho\right)$, which is then clearly equal to $\left(\begin{array}{c}2\lfloor d / \rho\rfloor \\ \lfloor d / \rho\rfloor\end{array}\right)$.

For the following equations, recall that $\delta_{a \mid b}$ is 1 if $a \mid b$ and 0 otherwise, and that $\mathrm{d}=$ $(r-1) / 2$. Also recall that when condition (3.7) is violated (as for some $r$ when $n=8$ ), we do not have a closed formula, and in such cases some instances of the function $A$ remain unevaluated (for certain values of $r$ ). 


$$
\begin{aligned}
A^{+}\left(r^{2}\right)= & \frac{1}{2}\left(\begin{array}{c}
2 d \\
d
\end{array}\right)+\frac{1}{2(2 d+1)} \sum_{1 \leq \rho \mid 2 d+1} \phi(\rho)\left(\begin{array}{c}
2\lfloor d / \rho\rfloor \\
\lfloor d / \rho\rfloor
\end{array}\right)^{2} \\
A^{+}\left(r^{4}\right)= & \frac{1}{6} d\left(\begin{array}{c}
2 d \\
d
\end{array}\right)^{4}+\frac{1}{2} d\left(\begin{array}{c}
2 d \\
d
\end{array}\right)^{2}+\delta_{3 \mid r} \frac{2}{3}\left(\begin{array}{c}
2\lfloor d / 3\rfloor \\
\lfloor d / 3\rfloor
\end{array}\right)\left(\begin{array}{c}
2 d \\
d
\end{array}\right) \\
A^{+}\left(r^{6}\right)= & \frac{36 d-1}{120} d^{2}\left(\begin{array}{c}
2 d \\
d
\end{array}\right)^{6}+\frac{3}{4} d^{2}\left(\begin{array}{c}
2 d \\
d
\end{array}\right)^{3}+\frac{1}{3} d\left(\begin{array}{c}
2 d \\
d
\end{array}\right)^{2}+\delta_{5 \mid r} \frac{4}{5}\left(\begin{array}{c}
2\lfloor d / 5\rfloor \\
\lfloor d / 5\rfloor
\end{array}\right)\left(\begin{array}{c}
2 d \\
d
\end{array}\right) \\
A^{+}\left(r^{8}\right)= & \frac{1}{2520}\left(2048 d^{3}-387 d^{2}+20 d-1\right) d^{2}\left(\begin{array}{c}
2 d \\
d
\end{array}\right)^{8}+\frac{1}{24}(32 d-5) d^{2}\left(\begin{array}{c}
2 d \\
d
\end{array}\right)^{4} \\
& +\frac{1}{4} d\left(\begin{array}{c}
2 d \\
d
\end{array}\right)^{2}+\delta_{7 \mid r} \frac{6}{7}\left(\begin{array}{c}
2\lfloor d / 7\rfloor \\
\lfloor d / 7\rfloor
\end{array}\right)\left(\begin{array}{c}
2 d \\
d
\end{array}\right)+\frac{4}{3} \delta_{r=3} \\
& +\frac{1}{4 r} \delta_{(r>3 \& 3 \mid r)} A\left((r / 3)^{2} r^{2} ; 4 r / 3\right) \\
A^{+}\left(r^{10}\right)= & \frac{1}{90720}\left(250000 d^{5}-100944 d^{4}+14167 d^{3}-1131 d^{2}+73 d-3\right) d^{2}\left(\begin{array}{c}
2 d \\
d
\end{array}\right)^{10} \\
& +\frac{1}{48}\left(125 d^{2}-30 d+1\right) d^{2}\left(\begin{array}{c}
2 d \\
d
\end{array}\right)^{5}+\frac{2}{5} d\left(\begin{array}{c}
2 d \\
d
\end{array}\right)^{2} \\
& +\delta_{9 \mid r} \frac{2}{3}\left(\begin{array}{c}
2\lfloor d / 9\rfloor \\
\lfloor d / 9\rfloor
\end{array}\right)\left(\begin{array}{c}
2 d \\
d
\end{array}\right)+\delta_{3 \mid r} \frac{(10 d-1)^{2}}{81}\left(\begin{array}{c}
2\lfloor d / 3\rfloor \\
\lfloor d / 3\rfloor
\end{array}\right)\left(\begin{array}{c}
2 d \\
d
\end{array}\right)^{3} .
\end{aligned}
$$

Notably, in the formulae above all rational functions of $d$ mentioned in Theorem 2 actually turn out to be polynomials. Computing for specific small values of $r$ gives the following table for the number $A^{+}\left(r^{n}\right)$ of unrooted $r$-regular maps with $n$ vertices. The entries "no formula" occur when $r$ is a proper multiple of an odd prime divisor of $n-2$, in which case we do not have a closed formula. Note that the missing values can be filled in, if required, by using Lemma 2 in a recursive computation for any given $h$. This allows one to compute the low coefficients of $M_{k}^{[h]}(x)$ for small $k$ and $h$, and thereby compute the numbers required for the remaining vertex-vertex cases in Theorem 1 (for any particular $r$ and $n$ ).

Table 3: Numbers of unrooted regular $n$-vertex maps, $2 \leq n \leq 20$, from closed formulae 


\begin{tabular}{rl}
\hline$r$ & $A^{+}\left(r^{2}\right)$ \\
\hline 3 & 2 \\
5 & 7 \\
7 & 39 \\
9 & 308 \\
11 & 3013 \\
13 & 33300 \\
15 & 394340 \\
17 & 4878109 \\
19 & 62232321 \\
21 & 812825244 \\
23 & 10818489817 \\
25 & 146250545528 \\
\hline
\end{tabular}

\begin{tabular}{rl}
\hline$r$ & $A^{+}\left(r^{4}\right)$ \\
\hline 3 & 6 \\
5 & 468 \\
7 & 80600 \\
9 & 16016560 \\
11 & 3360790440 \\
13 & 728936019504 \\
15 & 161858688461184 \\
17 & 36580777518027600 \\
19 & 8382066029146609800 \\
21 & 1941971956789550319920 \\
23 & 454006489072843947528288 \\
25 & 106944132919124515725427808 \\
\hline
\end{tabular}

\begin{tabular}{rl}
\hline$r$ & $A^{+}\left(r^{6}\right)$ \\
\hline 3 & 26 \\
5 & 111096 \\
7 & 513654400 \\
9 & 2243178389200 \\
11 & 9550256850912960 \\
13 & 40141310067406592352 \\
15 & 167483578096089845880576 \\
17 & 695587719666862982630642400 \\
19 & 2880023438585172541459822939200 \\
21 & 11898836836468227904716518897934240 \\
23 & 49083119653988050344170924762641714368 \\
\hline
\end{tabular}




\begin{tabular}{rl}
\hline$r$ & $A^{+}\left(r^{8}\right)$ \\
\hline 3 & 191 \\
5 & 39670362 \\
7 & 4742588317460 \\
9 & no formula \\
11 & 39758207592119720043060 \\
13 & 3253001744463113558023410456 \\
15 & no formula \\
17 & 19574639342029496912485843276022233800 \\
19 & 1467233390721250173975296095881808070004900 \\
21 & no formula \\
\hline
\end{tabular}

\begin{tabular}{rl}
\hline$r$ & $A^{+}\left(r^{10}\right)$ \\
\hline 3 & 1904 \\
5 & 17313263640 \\
7 & 53786496621600480 \\
9 & 115106032016630589567220 \\
11 & 204887911426306253833736206368 \\
13 & 326950476035695538194385010882728640 \\
15 & 485422668906114651402145718767396615062912 \\
17 & 684795515898773965920425882490399130420733638080 \\
\hline
\end{tabular}

\begin{tabular}{rl}
\hline$r$ & $A^{+}\left(r^{12}\right)$ \\
\hline 3 & 22078 \\
5 & 8581243954044 \\
7 & 696401295433093120200 \\
9 & 33156938554594811848607336600 \\
11 & 1210336263925368500658402392858157888 \\
13 & 37705498104552095463975261380309215629944592 \\
\hline
\end{tabular}

\begin{tabular}{rl}
\hline$r$ & $A^{+}\left(r^{14}\right)$ \\
\hline 3 & 282388 \\
5 & 4650485509103976 \\
7 & 9891028759926839453714800 \\
9 & no formula \\
11 & 7861944824653116454899492247205395134779280 \\
\hline
\end{tabular}




\begin{tabular}{rl}
\hline$r$ & $A^{+}\left(r^{16}\right)$ \\
\hline 3 & 3848001 \\
5 & 2692853733312152946 \\
7 & 150420303869075439022288355100 \\
9 & 3559090844072971395999935289096828404900 \\
11 & 54764349135521221379877401583610341415948624220820 \\
\hline
\end{tabular}

\begin{tabular}{ll}
\hline$r$ & $A^{+}\left(r^{18}\right)$ \\
\hline 3 & 54953996 \\
5 & 1641063780244320916056 \\
7 & 2410984386008752279281147366400400 \\
9 & 1273151076590084794217037825376103624331873200 \\
\hline
\end{tabular}

\begin{tabular}{ll}
\hline$r$ & $A^{+}\left(r^{20}\right)$ \\
\hline 3 & 814302292 \\
5 & 1041574815624514527248040 \\
7 & 40288343145550164807147826368004224240 \\
9 & no formula \\
\hline
\end{tabular}

For the reader's convenience, in Table 4 we represent the same numerical data for $r=3,5$ and 7 grouped by vertex degrees.

Table 4: Numbers of unrooted $r$-regular maps, $r=3,5,7$

\begin{tabular}{rrrr}
\hline$n$ & $A^{+}\left(3^{n}\right)$ & $A^{+}\left(5^{n}\right)$ & $A^{+}\left(7^{n}\right)$ \\
\hline 2 & 2 & 7 & 39 \\
4 & 6 & 468 & 80600 \\
6 & 26 & 111096 & 513654400 \\
8 & 191 & 39670362 & 4742588317460 \\
10 & 1904 & 17313263640 & 53786496621600480 \\
12 & 22078 & 8581243954044 & 696401295433093120200 \\
14 & 282388 & 4650485509103976 & 9891028759926839453714800 \\
16 & 3848001 & 2692853733312152946 & 150420303869075439022288355100 \\
18 & 54953996 & 1641063780244320916056 & 2410984386008752279281147366400400 \\
20 & 814302292 & 1041574815624514527248040 & 40288343145550164807147826368004224240 \\
\hline
\end{tabular}

\section{References}

[BenC94] E. A. Bender and E. R. Canfield, The number of degree restricted rooted maps on the sphere, SIAM J. Discrete Math. 7:1 (1994), 9-15. MR1259005 (94k:05093)

[Gao93] Z. C. Gao, The number of degree restricted maps on general surfaces, Discrete Math. 123 (1993), 47-63. MR1256081 (94j:05008) 
[GaoR97] Z. C. Gao and M. Rahman, Enumeration of k-poles, Annals of Combin. 1:1 (1997), 55-66. MR1474800 (98k:05085)

[Lis81] V. A. Liskovets, A census of non-isomorphic planar maps, Colloq. Math. Soc. J. Bolyai 25 (1981), Proc. Intern. Conf. "Algebr. Methods in Graph Th.", Szeged, Hungary, 1978, 479-494. MR0642058 (83a:05073)

[Lis85] V. A. Liskovets, Enumeration of nonisomorphic planar maps, Selecta Math. Sov. 4:4 (1985), 303-323 (Translation from two-part Russian original article published in: Geometric methods in problems in analysis and algebra, 106-117, 129, 132 and Problems in group theory and homological algebra, 103-115, Yaroslav. Gos. Univ., Yaroslavl', 1981). MR0709627 (84j:05057), MR0880223 (88c:05064)

[Lis98] V. Liskovets, Reductive enumeration under mutually orthogonal group actions, Acta Applic. Math. 52 (1998), 91-120. MR1649693 (2000b:05012)

[Lis04] V. A. Liskovets, Enumerative formulae for unrooted planar maps: a pattern, Electron. J. Combin. 11:1 (2004), RP 88, 14 pp. (electronic). MR2114192

[LisW87] V.A. Liskovets and T.R.S.Walsh, Ten steps to counting planar graphs, Congr. Numer. 60 (1987), 269-277. MR0945238 (89g:05057)

[Mul66] R. C. Mullin, On the average number of trees in certain maps, Canad. J. Math. 18:1 (1966), 33-41. MR0186581 (32 \#4040)

[Tut62] W. T. Tutte, A census of slicings, Canad. J. Math. 14:4 (1962), 708-722. MR0142470 (26 \#39)

[Wor81] N. C. Wormald, On the number of planar maps, Canad. J. Math. 33:1 (1981), 1-11. MR0608849 (83a:05074) 